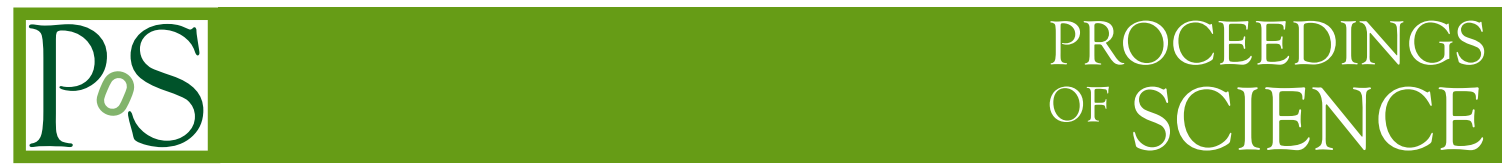

\title{
Analyticity and end-point behaviour of GPDs
}

\author{
Oleg Teryaev* \\ Bogoliubov Laboratory of Theoretical Physics, JINR, Dubna 141980, Russia \\ E-mail: teryaevetheor.jinr.ru
}

The QCD factorization for hard exclusive amplitudes is compared with their crossing and analytic properties. The crucial role is played by their mathematical structure described by Radon transform, leading to "holographic" property of GPDs at LO. The sensitivity of the representations based on analyticity and crossing to the behaviour of non-perturbative inputs near the boundary and critical points are analyzed. The bounds implied by crossing and analyticity for the angular distributions in two-photon processes are also discussed.

XVIII International Workshop on Deep-Inelastic Scattering and Related Subjects April 19 -23, 2010

Convitto della Calza, Firenze, Italy

${ }^{*}$ Speaker. 


\section{Introduction}

Exclusive hard processes described by Generalized Parton Distributions (GPD's) are the subject of extensive theoretical investigations for a few years (see e.g. [1,2] and Ref. therein). The present contribution is devoted to the important property of analyticity of such amplitudes and its implications. The crucial issue is the compatibility of analytical and crossing properties with QCD factorization which happens to be possible but non-trivial. The mathematical background is provided by integral geometry manifested in the form of Radon transform [3], while the new physical applications involve the angular distributions in hadron pairs two-photon production and the "breakup" GPDs describing, say, the hard exclusive processes with deuteron whose survival in its course is very unlikely.

Here we specifically address also the problem of the possible irregularities of GPDs near the boundary and other "critical" points, the notable example being suggested by the flat pion distribution amplitude (DA) [4, 5]. Note that the possible violation of QCD factorization may be bypassed [6] by the use of NPQCD methods relying on exact anomaly sum rule [7].

\section{Analyticity, crossing and GPDs near the critical points}

The leading order contribution of GPD's to the amplitudes of hard processes -DVCS and (longitudinal) vector meson production is described by the following integrals:

$$
\mathscr{H}(\xi)_{i}=\int_{-1}^{1} d x H_{i}(x, \xi)\left[\frac{1}{x-\xi+i \varepsilon} \pm \frac{1}{x+\xi-i \varepsilon}\right]
$$

where index $i$ describes the type of GPD, defining also the choice of \pm sign. The appearance of the same argument $\xi$ in the numerator and denominator results from the zero mass of the produced photon or its neglecting for vector meson. We also drop the dependence on the momentum transfer $t$. It is obvious, that only the (anti)symmetric part of GPD contributes, depending on that sign, which we will only consider in what follows, so that we will always discuss the integral,

$$
\mathscr{H}(\xi)=\int_{-1}^{1} d x \frac{H(x, \xi)}{x-\xi+i \varepsilon}
$$

dropping also the index $i$, as well as (anti)symmetrization index. This integral looks almost like the dispersion relation with respect to the variable $s$, where contribution of the crossed channel is usually taken into account by explicit addition of two terms in (2.1) and the reduction of the integration region to the positive $s$ (corresponding to positive $x$ ) only.

There is, however, the notable difference with the forward case, say, that of Deep Inelastic Scattering. Namely, the numerator depends also on $\xi$, which prevents from its direct identification as a spectral density. Nevertheless, the specific properties of $H$ as a Radon transform makes this dependence inessential.

Let us first consider the unphysical region $|\xi|>1$. Note, that the consideration of (2.1) in the unphysical region requires the appropriate analytical continuation of $H(x, \xi)$. As it was discussed in detail in [3], it is provided by the integration of double distribution over the straight lines with 
the "unphysical" slope:

$$
H(z, \xi)=\int_{-1}^{1} d x \int_{|x|-1}^{1-|x|} d y(F(x, y)+\xi G(x, y)) \delta(z-x-\xi y) .
$$

To identify this object with the physical quantity one should additionally consider the analytic continuation in $t^{1}$. The resulting object is just Generalized Distribution Amplitude (GDA) [10]. Note that the integration limits for $|\xi|>1$ are in fact $[-\xi, \xi]$ as follows from the spectral properties of double distributions. As a result, the unified expression for the DVCS and meson production amplitudes (in the common unphysical point $t=0$ ) takes the form:

$$
\mathscr{H}(\xi)_{i}=\int_{-\max (1, \xi)}^{\max (1, \xi)} d x H_{i}(x, \xi)\left[\frac{1}{x-\xi+i \varepsilon} \pm \frac{1}{x+\xi-i \varepsilon}\right]
$$

These limits were in fact implicitly assumed in $[3,11]$ as they are required to have the polynomiality for all $\xi$ which will be explored in a moment.

Note also that Radon transform properties are rather different in even- and od- dimensional spaces (see [3] and Ref. therein), so that the matrix elements involving the three external particles are of special interest. In particular, this corresponds to the GDAs for three pions production [12] and "breakup" GPDs, when, say, initial state deuteron does not survive in the hard reaction [?].

Note finally, that the so-called Polyakov-Weiss (PW) term [13], which originally did not emerge as a Radon transform, may be also included in such a form (and described by the function $G$ ), allowing its consideration in the unphysical region.

Let us consider (2.4) for $(\xi>1)$ and expand the denominator to get:

$$
\mathscr{H}(\xi)=-\int_{-\max (1, \xi)}^{\max (1, \xi)} d x \sum_{n=0}^{\infty} H(x, \xi) \frac{x^{n}}{\xi^{n+1}} .
$$

In the forward case, when one have instead of $H$ the forward distribution which does not depend on $\xi$, this series in the negative powers of $\xi$ (corresponding to positive powers of $s$ ) explicitly manifests the analyticity of $\mathscr{H}$. In the actual case of GPD's the key role is played by the mentioned polynomiality property: the moments of the function $H(x, \xi)$, namely the integrals in $x$ weighted with $x^{n}$, are polynomials of $\xi$ of power $n+1$. Therefore, the series is still containing only the non-positive powers of $\xi$, and the analyticity property is preserved.

The proven analyticity in the unphysical region allows now to write the standard dispersion relation instead of (2.2):

$$
\mathscr{H}(\xi)=\int_{-1}^{1} d x \frac{H(x, x)}{x-\xi+i \varepsilon}
$$

Note that the integration in this equation runs again from -1 to 1 provided the singulatity of double distribution at the edge point $(0,1)$ is absent, so that the resulting expressions may be used for the check of that. This formula represents the holographic property of GPD: namely, the full information about, say, DVCS amplitude in the considered leading approximation is contained in the one-dimensional section $x=\xi$ (related, by the symmetry properties to $x=-\xi$ ) of the two dimensional space of $x$ and $\xi$. In what follows we will study the relations of dispersion representation

\footnotetext{
${ }^{1}$ The account for finite $t$ effect in he dispersion relation for DVCS requires a special care [8,9].
} 
with the standard factorization formula. Let us consider the difference of (2.6) and (2.2):

$$
\Delta \mathscr{H}(\xi) \equiv \int_{-1}^{1} d x \frac{H(x, x)-H(x, \xi)}{x-\xi+i \varepsilon}=\sum_{n=1}^{\infty} \frac{1}{n !} \frac{\partial^{n}}{\partial \xi^{n}} \int_{-1}^{1} H(x, \xi) d x(x-\xi)^{n-1}=\text { const } .
$$

The emerging constant term is by no means strange and is nothing else than a subtraction constant. It is generated by the maximal powers in $\xi$, provided by PW terms. To quantify this important relation, let us calculate $\Delta \mathscr{H}(\xi)$, by substituting the definition (2.3):

$$
\Delta \mathscr{H}(\xi)=\int_{-1}^{1} d x \int_{|x|-1}^{1-|x|} d y \frac{G(x, y)}{1-y},
$$

where we used the following property of delta functions in (2.3): $\delta(z(1-y)-x)=\delta(z-x /(1-$ $y)) /(1-y)$ (as $|1-y|=1-y$ in the integration region), while integrating the $H(z, z)$ term. This proof does not require the existence of infinite number of derivatives of $H$ and shows that the very existence of double distribution is sufficient to justify the holographic property. so it should be stable against at least LO QCD corrections. As we see, only the $G$ function leads to the finite subtraction. This provides an extra justification for the original form of PW term, when it resides in the ERBL region $|x|<\xi$. In that case it is obvious, that it provides no contribution to the imaginary part of DVCS amplitude, and is reduced to finite subtraction constant:

$$
\int_{-\xi}^{\xi} d x \frac{D(x / \xi)}{x-\xi+i \varepsilon}=\int_{-1}^{1} d z \frac{D(z)}{z-1}=\text { const }
$$

In particular, the amplitude in the GDA kinematical region $\xi>1$ may be represented in the form (recall that the regular behaviour at the edge point is required)

$$
\begin{aligned}
& \mathscr{H}(\xi)=-\int_{-1 / \xi}^{1 / \xi} d x \sum_{n=0}^{\infty} H(x, \xi) \frac{x^{n}}{\xi^{n+1}} \\
& =-\int_{-1 / \xi}^{1 / \xi} d x \sum_{n=0}^{\infty} H(x, x) \frac{x^{n}}{\xi^{n+1}}+\Delta \mathscr{H} .
\end{aligned}
$$

It is clearly seen, that while the same subtraction constant as in DVCS channel appears, the coefficients of the higher powers of $1 / \xi$ which, in turn, correspond to higher powers of scattering angle, are defined by the higher moments of $H(x, x)$. The latter may be bounded from below by the moments of forward distribution $H(x, 0)$. This may be qualitatively understood, as the integration line in $H(x, x)$ is much longer (for large $x$ ) and lies to the left of that in $H(x, 0)$. From the more formal point of view, one may recast the positivity constraints for parton distributions $[1,14]$ in the form

$$
H(x, x)<\operatorname{const} \sqrt{H(x, 0)},
$$

where the $x$ - independent constant is defined by the photoabsorbtion cross-section for a given quark flavour and the transitional momentum transfer $Q_{0}$ (c.f. [15] ) where leading twist approximation is still applicable. Passing to the limit $x \rightarrow 1$ one may keep only the proton intermediate state in the derivation of the positivity constraint which saturates it. As a result, the decrease power $\beta$ of $H(x, x) \sim(1-x)^{\beta}$ for $x \rightarrow 1$ is twice smaller than for $H(x, 0)$. 
This puts the lower bound for the decrease of the coefficient of higher powers of c.m. scattering angle in (2.10). One should note that this decrease is much slower for pion pair production than for nucleon one, because of slower decrease of (forward) pion distribution due to the quark counting rules. In general, the angular distribution in $t$-channel is determined by the threshold behaviour in $s$-channel.

Note also that this bound for pion may be relevant for the shape of pion DA, as combining the $\xi \rightarrow 1$ limit of (2.4) and (2.7) (with no subtraction in isovector channel) with soft pion theorem, one may relate GPD at the critical point $(x, x)$ to DA.

Conversely, the finite value of GPD at this point (for pion or maybe proton) being the natural generalization of flat pion DA, may result in the logarithmic growth of the subtraction term with $Q^{2}$ similar to that of pion transition formfactor.

\section{Conclusions}

The analytical and crossing properties of hard exclusive processes involve a beautiful mathematics of integral geometry and has a numerous physical implications, in particular, related to important problems of GPDs endpoint behaviour.

I am indebted to organizers and conveners, and in particular to Simonetta Liuti, for possibility to present the talk on which this paper is based. This work was supported in part by the Russian Foundation for Fundamental Research, (grants 09-02-01149 and 09-02-00732).

\section{References}

[1] A. V. Belitsky and A. V. Radyushkin, Phys. Rept. 418, 1 (2005) [arXiv:hep-ph/0504030].

[2] S. Boffi and B. Pasquini, Riv. Nuovo Cim. 30, 387 (2007) [arXiv:0711.2625 [hep-ph]].

[3] O. V. Teryaev, Phys. Lett. B 510, 125 (2001).

[4] A. V. Radyushkin, Phys. Rev. D 80, 094009 (2009) [arXiv:0906.0323 [hep-ph]].

[5] M. V. Polyakov, JETP Lett. 90, 228 (2009) [arXiv:0906.0538 [hep-ph]].

[6] Y.N. Klopot, A.G. Oganesian, O.V. Teryaev, work in progress.

[7] J. Horejsi and O. Teryaev, Z. Phys. C 65, 691 (1995).

[8] D. Drechsel, B. Pasquini and M. Vanderhaeghen, Phys. Rept. 378, 99 (2003) [arXiv:hep-ph/0212124].

[9] G. R. Goldstein and S. Liuti, Phys. Rev. D 80, 071501 (2009) [arXiv:0905.4753 [hep-ph]].

[10] M. Diehl, T. Gousset, B. Pire and O.V. Teryaev, Phys. Rev. Lett. 81 (1998) 1782.

[11] I. V. Anikin and O. V. Teryaev, Phys. Rev. D 76, 056007 (2007) [arXiv:0704.2185 [hep-ph]].

[12] B. Pire and O. V. Teryaev, Phys. Lett. B 496, 76 (2000) [arXiv:hep-ph/0007014].

[13] O. V. Teryaev, AIP Conf. Proc. 1056, 130 (2008).

[14] M.V. Polyakov and C. Weiss, Phys. Rev. D60 (1999) 114017.

[15] X. Artru, M. Elchikh, J. M. Richard, J. Soffer and O. V. Teryaev, Phys. Rept. 470, 1 (2009) [arXiv:0802.0164 [hep-ph]].

[16] J. Soffer and O. Teryaev Phys. Rev. Lett. 70, 3373 (1993); Phys. Rev. D 70, 116004 (2004) [arXiv:hep-ph/0410228] 